Dear session organizers,

authors thanks the reviewers for the very useful suggestion and comments to include the quality of the paper. The text was modified accordingly and changed parts were highlighted with the red color. A detailed response to reviewer comments follows.

\title{
Response to Reviewer 1
}

Stochastic Eulerian field PDF methods are combined with a combination of acceleration strategies to reduce the computational cost associated with the use of large chemical mechanisms in CFD. The resulting model is used to simulate autoignition in a high-pressure turbulent spray combustion vessel under diesel-engine-like conditions. While the individual methods are not new, the combination of methods and their application to autoignition for enginerelevant conditions is novel, and would be of interest to the SAE audience. This is an approach that has significant potential for accurately capturing effects of turbulence-chemistry interaction over a wide range of engine operating conditions.

Authors thanks the reviewer for the comments. The proposed combustion model based on Eulerian Stochastic fields will be continuosly developed and applied soon to more realistic engine studies.

\section{Response to Reviewer 2}

In the work described in this manuscript, the authors pursued to bring a Stochastic Eulerian Field (SEF) PDF approach into IC engine combustion CFD analysis. The work was performed using OpenFOAM CFD code - an open source code. The authors exercised the modeling for one of the ECN database cases (i.e., 15After having read through the whole content of the manuscript, this reviewer considers the work is incomplete, suggesting the authors to re-submit it in 2015 SAE Congress after improving the quality of the work.

The title of the paper "Towards the Use of Eulerian Field PDF Methods for Combustion Modeling in IC Engines" should clearly indicate that this is a first attempt to apply what is considered by the combustion community to be one of the most complex and realistic models currently available to predict flame propagation. Presentation of preliminary results and discussion about them within the SAE world congress toghether with the major experts of the engine combustion community represents, to the authors' opinion, one of the best ways to understand the main advantages and drawbacks of such model and to find proper ways to improve it. For this reason, authors do not think this work is incomplete but it represents an important step towards the adoption of advanced models to predict combustion in IC engines.

1. The amount of the work is insufficient The authors began with an opening of good cause in Abstract: "Detailed chemistry and turbulence-chemistry interaction need to be properly taken into account for a realistic combustion simulation of IC engines where involve a a wide range of combustion regimes and require a proper description of several phenomena such as auto-ignition, flame stabilization, diffusive combustion and lean premixed flame propagation." However, the authors themselves agreed that "Further work is required to include a comprehensive validation at different ambient conditions and investigate if it is possible to reduce the number of stochastic fields required for the statistical convergence." at the end of the manuscript.

This reviewer's comment is not completely clear to the authors. The first sentence is just a general consideration, while the last one clearly states that this is just a preliminary work and for this reason further validation is required.

2. The model description is poor This reviewer has only moderate level of knowledge on the SEF PDF method. However, after reading the manuscript, the reviewer becomes skeptical of the authors' model implementation into OpenFOAM, for the difficulty of following the content in "Numerical Models". For example, on Page 3, the paragraph following Eq. (1), "Here summation is implied", does not seem to comment on Eq. (1). Further, what is $N_{-} F$ in the next paragraph? How to understand it? Further more, what is the Wiener term in Eq. (2)? What is the vector-related Wiener process? And, Does $W$ in Eq. (4) share the same meaning as W in Eq. (2)? If NOT, why does the author choose a way of expression confusing the reader? Is S_alpha in Eq. (5) different from omega in Eq. (4)?

For what concerns the model description, in this work it was not possible to include all the complete details about 
how stochastic combustion models and related processes work. We recommend the reviewer to look at references [21-28] for further information. Eq. 1 illustrate the generic formulation of a transport equation for the joint pdf function inside the computational domain. Such equation is too complex to be solved directly (it is a transport equation for a function and not a scalar or vector field) and for this reason it is solved either with the help of Lagrangian particles (Lagrangian PDF methods) or by using Stochastic fields. The statement "summation is implied ..." is referred to divergence terms appearing in Eq. 1 and other transport equations included in this work as well. The Wiener vector is a stochastic source term which is introduced in each stochastic field transport equation to generated a consequent PDF for enthalpy and each chemical species involved. This aspect was clarified in the text and highlighted with red color. Authors also thanks the reviewer for the other observations and text was changed accordingly, in particular:

- the same notation was adopted for all the Wiener terms, avoiding confusion

- same expression for chemical source term was also used (omega and not S_alpha).

3. No deep-dive discussion with the results of the study On Page 5, "the importance of the Wiener term in Eq.(2)" is emphasized by the author. Irrespective of the importance of other terms in Eq. (2), the author identified four challenges associated. For example, in the 1st paragraph of "Experimental Validation", the authors mentioned that "in case of spray simulation, computed fuel mass from stochastic fields might not coincide with what was really evaporated and, consequently, a wrong prediction is expected for both fuel-air mixture formation and combustion processes." However, the author just simply presented Figure 3 and Figure 4 with no deep-dive discussion. The authors concluded that, by means of Table 3 or Figure 4(b), using 32 fields could ensure a good solution. But, the reviewer is not very much convinced (see, Table 3).

Most of the Experimental Validation paragraph is dedicated to the model performance at non-reacting conditions. This choice was mainly done in order to understand if the stochastic field method is able to properly predict variances and how it performs for what concerns conservation of instantaneous fuel mass. Such quantity was considered quite important since it mainly governs at reacting conditions the heat release rate profile. When performing a sensitivity analysis, it was found that at least 32 fields are required to achieve:

- proper conservation of instantaneous fuel mass

- realistic and symmetric profiles of mixture fraction and its variance

More comments were added in that part of the text to better clarify why 32 stochastic fields can ensure a good solution.

4. The scope is not well defined The authors laid out three tasks for the study on Page 5-6. Task 3 is defined as "comparison between predicted pressure trace and flame structure between SEF, well-stirred reactor and mRIF model." This reader considers Task 3 should be the core motivating the study. However, the authors spent a great amount of effort on the first two tasks but only briefly mentioned this aspect, with one figure (Figure 9). It is unclear why no comparison with the experimental visualization (from ECN database) is provided.

Authors thanks the reviewer for such observation. Assessment of the model at non-reacting conditions required a significant amount of time and for this reason it was not possible, by the deadine date of the paper, to also include a detailed validation of the model at non-reacting conditions. Hence, only a flame structure comparison between the three tested models was performed and a detailed comparison between them, also including different operating conditions will be carried out in a future work. However, authors think that the investigation proposed already include a significant amount of results.

\section{Response to Reviewer 3}

This paper presents an interesting PDF methods for turbulent combustion modeling, and is very well written. I recommended paper to be approved and for journal publication. Some comments for the authors to address and suggestions for the authors to refine their paper as below 
Authors thanks the reviewer for the general comments. Paper was modified following suggested changes.

(1) Eq. (3) and (7) seem inconsistent

Equation (7) was corrected, since it shows the calculation of the fuel mass and not fuel mass fraction.

(2) Figure 4 compared total evaporated mass for different number of fields. It is not clear why the authors concern about those. They are expected to be different as different average mixture fraction is predicted resulting in different evaporation profile. I will be more concerned on fuel mass conservation as total (i.e. how much liquid mass reduced, how much vapor mass increased)

Authors thanks the reviewer for this consideration. As stated in the text, the evaporation source term is the same for any of the stochastic fields and spray evolves according to the average composition in each cell. Figure 4 reports the total amount of fuel mass.

(3) The paragraph prior to CPU time reduction is not clear. More detail such as EMST can help the reader to understand

Text was changed including further details on mixing models and requirements about them from the SEF method.

(4) It is difficult to follow 1st paragraph of experimental validation.

1st paragraph was partially rewritten to make the contents more clear

(5) How evaporation source term in Eq (2) is modeled

Evaporation source term is computed according to the average composition in each cell. Then such quantity is applied to the vapor fuel species of any stochastic field. 


\title{
Towards the Use of Eulerian Field PDF Methods for Combustion Modeling in IC Engines
}

\author{
Author, co-author list (Do NOT enter this information. It will be pulled from participant tab \\ in MyTechZone) \\ Affiliation (Do NOT enter this information. It will be pulled from participant tab in MyTechZone)
}

Copyright (C) 2014 SAE International

\begin{abstract}
Detailed chemistry and turbulence-chemistry interaction need to be properly taken into account for a realistic combustion simulation of IC engines where advanced combustion modes, multiple injections and stratified combustion involve a wide range of combustion regimes and require a proper description of several phenomena such as auto-ignition, flame stabilization, diffusive combustion and lean premixed flame propagation. To this end, different approaches are applied and the most used ones rely on the wellstirred reactor or flamelet assumption. However, wellmixed models do not describe correctly flame structure, while unsteady flamelet models cannot easily predict premixed flame propagation and triple flames. A possible alternative for them is represented by transported probability density functions (PDF) methods, which have been applied widely and effectively for modelling turbulent reacting flows under a wide range of combustion regimes. For IC engine simulations, the most promising ones are the Eulerian field PDF methods (SEF) whose formulation was originally proposed by Valiño and Sabel'nikov. Such models can be easily incorporated into CFD codes and are less computationally intensive with respect to Lagrangian approaches. In particular, Lagrangian particles are replaced by stochastic fields and transport equations are solved for them including a random process as a source term. Purpose of this work is the assessment of a SEF combustion model, that has been implemented into the Lib-ICE code, which is based on the OpenFOAM technology. To make the use of detailed chemistry possible in a reasonable amount of time, a multi-zone approach was incorporated in the combustion model and coupled with the TDAC technique, combining in-situ adaptive tabulation and dynamic adaptive chemistry. Experimental validation was carried out by simulating Diesel combustion ex-
\end{abstract}

periments at constant volume conditions.

\section{Introduction}

The continuous innovation towards the achievement of more efficient and less polluting engines has significantly extended the range of occurring combustion regimes and transitions from one mode to another in the same engine technology. Kinetically controlled combustions with or without spark-ignition such as $\mathrm{HCCl}, \mathrm{PCCl}$ or SACl $[1,2,3]$, Diesel engines with multiple injections and/or high EGR [4], GDI engines operating with a lean stratified charge $[5,6,7]$ are examples of such a trend. On the modeling point of view, this evolution is motivating the definition of a unique and high fidelity combustion models that should be applicable in all relevant combustion regimes [8]. With such a premise, it is clear the need to incorporate in the proposed model a detailed chemistry approach, but also to account at some stage of the non-linear interaction between fluid mixing and finiterate chemistry. On the other hand, in literature there are many studies $[9,10,11,12]$ in which successful simulations of Diesel, $\mathrm{HCCl}$ and premixed combustions were achieved by neglecting any sub-grid interaction between turbulence and chemistry, especially emphasis was give on the capability of well predicting auto-ignition rather than the flame structure. In this sense, very common alternative approaches, mainly for Diesel combustion simulations are the multiple Representative Interactive Flamelet Model (mRIF) and the Conditional Moment Closure (CMC). The first approximates the flame structure as a set of multiple unsteady laminar diffusion flames (flamelets). Their evolution is computed in the mixture fraction space [13] where species and energy equations are solved. Effects of mixing are incorporated in the scalar dissipation rate, which is com- 
puted as a conditional average of its distribution in the CFD domain. Use of multiple flamelets ensures a better prediction of both flame structure and autoignition, since spatial variations of the scalar dissipation rate are properly taken into account [14]. The CMC model introduces a further level of detail, since flamelet equations are solved including also a term accounting for flow-field effects. This requires a simplified 1D, 2D or coarse 3D mesh of the studied geometrical domain, and suitable mapping techniques to interpolate computed solution on the real mesh. CMC model is very powerful, but computationally very demanding due to the need to solve flamelet equations directly in the CFD domain [15, 16]. For this reason its application is limited to small chemical mechanisms operating only with simplified surrogate fuels, like n-heptane [17]. Incorporating detailed chemistry in premixed or partially-premixed combustion is more complicated since flame propagation takes generally place in a thin wrinkled laminar flame front. Application of the flamelet concept to SI combustion (premixed or stratified) is not immediate, due to the need to properly account for both ignition process and pressure effects in post-flame kinetics governing both soot and $\mathrm{NO}_{x}$ formation. Finally, the third possible approach to model turbulent combustion, accounting for multi-regime conditions, is based on the application of probability function methods (PDF), which offer compelling advantages for modeling chemically reacting turbulent flows, providing an effective resolution to the closure problems that arise from averaging the highly nonlinear chemical source terms. Most developments of PDF models derive from Pope's original work [18] and are extensively discussed in [19, 20]. A comprehensive review and a perspectives on recent advances and trends can be found in [21, 22]. In a composition PDF method, a modelled transport equation is solved for the one-point, one-time joint PDF of the composition variables describing the local thermochemical state of the reacting system. The main advantage of PDF methods is that the chemical source term appears in closed form. Multiple strategies were proposed to solve PDF transport equations: most of the work was carried out by Lagrangian particle Monte Carlo methods where the PDF is represented by a large number of notional particles that evolve according to stochastic equations, and local mean quantities are estimated as appropriately weighted averages over the particles in a small neighborhood. However, implementation of Lagrangian particle methods into conventional CFD codes is complex and consistency issues might arise [21]. Moreover, a large number of particles is required to simulate statistically non-homogeneous systems, and particle-based PDF methods are computationally demanding. Examples of applications of Lagrangian PDF methods for IC engine simulations can be found in [23, 24]. A possi- ble alternative is represented by Stochastic Eulerian Field PDF (SEF) methods, which might be more compatible and efficient when implemented into conventional CFD codes. Valiño presented such approach in [25], demonstrating its equivalence with Lagrangian methods. Examples of application of the SEF to spray combustion [26, 27] , turbulent non-premixed [28] and premixed combustion [29] can be found in literature. In this work, a novel methodology to simulate modern IC engine combustion with Stochastic Eulerian Field PDF method and detailed chemistry is presented. The SEF method was implemented into the LibICE code, based on the OpenFOAM@technology and extensively applied in the past for simulations of both premixed and non-premixed combustion in IC engines [30, 31, 32]. In particular, a novel, fully parallelized technique for on-line chemistry tabulation was employed, integrating the chemistry in a very limited set of points. Computational overheads were limited to the solution of a large number of transport equations which are multiple of the number of adopted stochastic fields. The proposed methodology was assessed and validated simulating Diesel combustion experiments. First, non reacting conditions were simulated to understand how the model predicts the mixture fraction distribution and its variance. Then combustion simulations were carried out and the flame structure computed by the SEF model was compared with the ones from well-mixed and the multiple RIF approaches.

\section{Numerical models}

\section{Stochastic Eulerian field (SEF) PDF method}

In an open, ideal-gas, single-phase, multi-component reacting mixture containing $N_{s}$ chemical species, the local thermochemical state and the species chemical production rates $\Omega$ can be determined from the $N_{s}$ species mass fractions $Y$ and the mixture specific enthalpy $h$. This set of variables will be referred to as composition variables denoted by $\phi$ a vector whose dimension is $N_{\phi}=N_{s}+1$. The corresponding composition joint PDF is denoted by $f_{\phi}=f_{\phi}(\psi ; \mathbf{x}, t)$. Conventional (denoted using angled brackets, \langle\rangle ) and mass-weighted or Favre-averaged (denoted using a tilde, $\sim$ ) mean values of any function of the composition variables, $Q=Q(\phi)$, can be expressed as integrals of the PDF over its sample space.

The starting point is a modelled transport equation for the joint PDF of the $N_{s}$ species mass fractions $Y$ the 
mixture specific enthalpy $h$ :

$$
\begin{gathered}
\frac{\partial \rho f_{\phi}}{\partial t}+\frac{\partial \rho \tilde{u}_{i} f_{\phi}}{\partial x_{i}}+\frac{\partial \rho \Omega_{\alpha} f_{\phi}}{\partial \psi_{\alpha}}= \\
\frac{\partial}{\partial x_{i}}\left[\Gamma_{T \phi} \frac{\partial\left(\rho f_{\phi} /\langle\rho\rangle\right)}{\partial x_{i}}\right]+\frac{1}{2} \frac{\partial\left[C_{\phi} \omega\left(\psi_{\alpha}-\tilde{\phi}_{\alpha} \rho f_{\phi}\right)\right]}{\partial \psi_{\alpha}}
\end{gathered}
$$

Here summation is implied over repeated Roman (Cartesian coordinate index) or Greek (composition variable index) subscripts within a term. Transport in physical space by the mean velocity $\tilde{u}_{i}$ and transport in composition space by chemical reaction $\Omega_{\alpha}$ appear in closed form on the left-hand of Eq. 1. The first term on the right-hand side corresponds to a gradient transport model for turbulent velocity fluctuations, and the second term corresponds to an interaction-byexchange-withthe-mean (IEM) model [33] for molecular transport or mixing.

In the stochastic Eulerian field (SEF) PDF method, $N_{F}$ notional Eulerian fields evolve according to stochastic PDFs. The system of stochastic PDEs is designed such that its one-point, one-time Eulerian joint PDF evolves according to Eq. 1. The number of PDEs to be solved is equal $N_{\phi} \times N_{F}$, and standard Eulerian CFD algorithms are employed to solve stochastic PDEs of the method [28]. In this work, the SEF method was implemented following the approach introduced by Valiño where the SEF equation corresponding to Eq. 1 is written as:

$$
\begin{gathered}
d\left(\langle\rho\rangle \phi_{\alpha}^{\#}\right)= \\
-\frac{\partial\langle\rho\rangle \tilde{u}_{i} \phi_{\alpha}^{\#}}{\partial x_{i}} d t+\Omega_{\alpha}\left(\phi^{\#}\right) d t+S^{\#} d t \\
-\frac{1}{2} C_{\phi} \omega\left(\phi_{\alpha}^{\#}-\tilde{\phi}_{\alpha}\right) d t \\
+\frac{\partial}{\partial x_{i}}\left[\Gamma_{T \phi} \frac{\partial \phi_{\alpha}^{\#}}{\partial x_{i}}\right] d t+\left(\frac{2 \Gamma_{T \phi}}{\langle\rho\rangle}\right)^{1 / 2} \frac{\partial \phi_{\alpha}^{\#}}{\partial x_{i}} d W_{i}^{\#}
\end{gathered}
$$

the superscript \# refers to any one of the fields in the vector $\phi ; S^{\#}$ is the source term due to spray evaporation, different for each species but identical for each stochastic field; $\omega$ is the turbulence mixing frequency defined as the ratio between turbulent kinetic energy $k$ and dissipation rate $\varepsilon$. $\mathbf{W}^{\#}$ denotes a vector-valued Wiener process that varies in time, but is independent of spatial location. Purpose of the Wiener vector is to introduce a stochastic noise in transport equations and generate a consequent PDF for enthalpy and each chemical species. The last two terms on the right hand side of Eq. 2 involve the apparent turbulent diffusivity $\Gamma_{T \phi}$ and correspond to a gradient transport model for turbulent velocity fluctuations.
In the absence of the Wiener term $\mathbf{W}^{\#}$, Eq. 2 is equivalent to a PDE. However, the stochastic field equations are written in increment form to emphasize that the stochastic term is not differentiable with respect to time. A key point in the Valiño formulation is that each field must be smooth (twice continuously differentiable) at the scale of the computational mesh. To be consistent with this statement, the same random increment is applied uniformly in each coordinate direction for each field by approximating $\mathbf{W}^{\#}$ at the beginning of each computational time-step and to treat explicitly the last term on the rhs in Eq. 2. Following earlier SEF modelling studies, the $d W_{i}^{\#}$ is approximated as $d t^{1 / 2} \eta_{i}$, where $\eta_{i}$ is a $\{-1,+1\}$ dichotomic vector. An operator-splitting strategy is used for the chemical and spray source terms, the mixing term and the random term in Eq. 2. Density-weighted mean quantities are then obtained by ensemble averaging over the fields:

$$
\tilde{Q}=\tilde{Q}(\mathbf{x}, t) \approx \frac{1}{N_{F}} \sum_{n=1}^{N_{F}} Q\left(\phi^{n}(\mathbf{x}, t)\right)
$$

An important difference between Lagrangian particle and SEF methods is that, in the former, turbulent transport is represented by a stochastic term in physical space, while in the latter, turbulent transport is represented in part by a stochastic term in composition space (Eq. 2). This can result in unphysical field compositions, and measures must be taken to minimize these excursions. Here the maximum magnitude of the stochastic term in the species equations is limited to the difference between the current value of the species mass fraction and the nearest physical bound on the species (either zero or unity) [34]. Despite very promising, Eulerain field PDF methods require specific developments mainly for what concerns the possible mixing models that can be adopted. In particular, the spatial smoothness has to be preserved and this aspect imposes some restrictions in case of models having stochastic processes with jumps, like the Curl's model. Another very promising model is the EMST, where the change of particle composition is determined by particle interactions along the edges of a Euclidean Minimum Spanning Tree constructed in composition space. However, implementation of the EMST model into the Eulerian stochastic method requires proper development efforts and detailed verifications about its assumptions. For all such reasons, the IEM (Interaction by Exchange with the Mean) mixing model was used in this work, since it ensures spatial smoothness. So far, SEF methods were only applied to model simplified configurations and, due to the need to use a large number of stochastic fields to properly represent the PDF distri- 
bution of the chemical species involved, small mechanisms were generally adopted to achieve results in a reasonable amount of computational time.

\section{CPU time reduction}

To compute chemical reaction rates in Eq. 2, an operator splitting technique is used. In particular, an ODE stiff solver takes the thermodynamic conditions (composition and temperature) of each stochastic field in any cell and integrates the chemical problem within the time-step, solving the species and energy equations. Then species mass fractions are updated as:

$$
\phi_{\alpha}^{\# *}(t+\Delta t)=\phi^{\#}(t)+\int_{t}^{t+\Delta t} \dot{\omega}^{\#} \frac{W^{\#}}{\rho_{\alpha}} d t^{\prime}
$$

where $\rho_{\alpha}$ is the density related to a stochastic field, $\dot{\omega}^{\#}$ is the reaction rate and $W^{\#}$ is the molecular weight of the chemical species. Solution of Eq. 4 is carried out by means of a multi-step, Semi-Implicit Bulirsch-Stoer method, SIBS [35].

Finally, the reaction rate $\Omega_{\alpha}$ is estimated as:

$$
\Omega_{\alpha}=\frac{\phi_{\alpha}^{\#^{*}}(t+\Delta t)-\phi_{\alpha}^{\#}(t)}{\Delta t}
$$

and it is included in the chemical species and enthalpy transport equations as a source term. Directintegration of chemistry introduces significant computational overheads in the simulation when SEF methods are used. As a practical example, the use of 20 stochastic fields in a Diesel combustion simulation ( 20000 cells during main combustion) and a 100 species mechanism will require to integrate 40 millions of stiff equations for each CFD time step taking time-scales of the $0.1-10 \mu \mathrm{s}$ order into account. This is not feasible and for this reason solutions to reduce the CPU time need to be found. To this end, two different approaches are generally followed: in the first one, chemical composition or reaction rates are retrieved from large tables including pre-computed reaction rates or flamelet solutions $[36,37,38,39]$ while the second approach computes on-line the chemical species reaction rates by means of stiff integrators $[40,30,41]$. Tabulated kinetics make simulations very fast mainly when applied to constant-pressure conditions. However, the need to include pressure, equivalence ratio, progress variable and EGR variations in them grows their size significantly and makes their application complicated due to the need to load, store and retrieve a very large amount of data even shared by different processors.
Possible recent improvements were proposed in [42]. When using direct-integration, CPU time can be drastically reduced by the use of on-line techniques for mechanism reduction and tabulation $[43,44,30,45]$ preserving the accuracy of the results and the flexibility of the method with respect to the adopted kinetic mechanism. In this work, a novel parallel methodology was developed which combines three different chemistry acceleration techniques: a multi-zone method, known as Chemistry Coordinate Mapping (CCM), Dynamic Adaptive Chemistry (DAC) and InSitu Adaptive Tabulation (ISAT).

To integrate the stiff ODEs of elementary reactions and estimate the source term in the species transport and energy equations for each stochastic field, the CCM method works as follows [31, 45]: first, a phase space consisting of three principal variables $\tilde{T}$, $J_{H}$ and $\beta=\log _{10}\left(\nabla J_{H} \cdot \nabla J_{H}+1\right)$ is constructed. $J_{H}$ is elemental mass fraction of hydrogen atom defined as:

$$
J_{H}=\sum_{k=1}^{N} \frac{W_{H}}{W_{k}} \beta_{H, k} \tilde{Y}_{k},
$$

where $W_{H}$ and $W_{k}$ are the atomic and molecular weights of the hydrogen and the $k$-th species, respectively. $\beta_{H, k}$ is the number of $\mathrm{H}$-atoms in the $k$-th species. Note that variables $\beta$ and $\nabla J_{H} \cdot \nabla J_{H}$ are uniquely related each other. In this way, for each stochastic field, computational cells are mapped into a three dimensional $\left(T, \nabla J_{H}, \beta\right)$ space.

Let the phase space be discretized by $N_{T}$ number of temperature zones, $N_{J}$ number of $J_{H}$ zones, and $N_{\beta}$ number of $\beta$ zones. The $(i, j, k)$ cell in the physical domain is mapped to the $(l, m, q)$ zone in the $\left(\tilde{T}, J_{H}, \beta\right)$ space. In discretized form the mapping is between the index $(i, j, k)_{\alpha}$ of the cells for each stochastic field in the physical domain to the index $(l, m, q)$ of the zones in the phase space. For each cell in the physical domain, cell indices, i.e., the value of $i_{T}(i, j, k)$, $i_{J}(i, j, k)$, and $i_{\beta}(i, j, k)$ in the phase space are stored on line at each time-step during the simulation, and they will be used later for the procedure of mapping back results from the phase space to physical space. The mean values of the variables in the phase space zones are determined and used as the initial condition for integrating the reaction rates. The mean reaction rate is then computed for each zone and mapped back to the cells in the physical space using the stored mapping index. Differently from many of the available multi-zone approaches [46, 47], using only a two-dimensional tabulation that accounts for mixture fraction and temperature, in the CCM approach local flow and mixing conditions are also taken into account through the $\nabla J_{H} \cdot \nabla J_{H}$ term. This makes the proposed tabulation method more oriented to the 
combustion mode that is going to be simulated. CCM reduces the computational time since the size of the $\left(\tilde{T}, J_{H}, \beta\right)$ space where chemistry equations are integrated is generally 1-2 orders of magnitude lower than the number of employed CFD cells. Furthermore, compositions from all stochastic fields are mapped onto a single $\left(\tilde{T}, J_{H}, \beta\right)$ space and then integration is performed on multiple processors, with each one of them receiving approximately the same number of points to be integrated with almost the same range of thermodynamic conditions. In this way, different decomposition methods are used between flow solution and chemistry integration, with this last one performed with a very good load balancing on a relatively small number of points. Application of parallel CCM to chemistry integration with SEF model is displayed in Fig. 1.

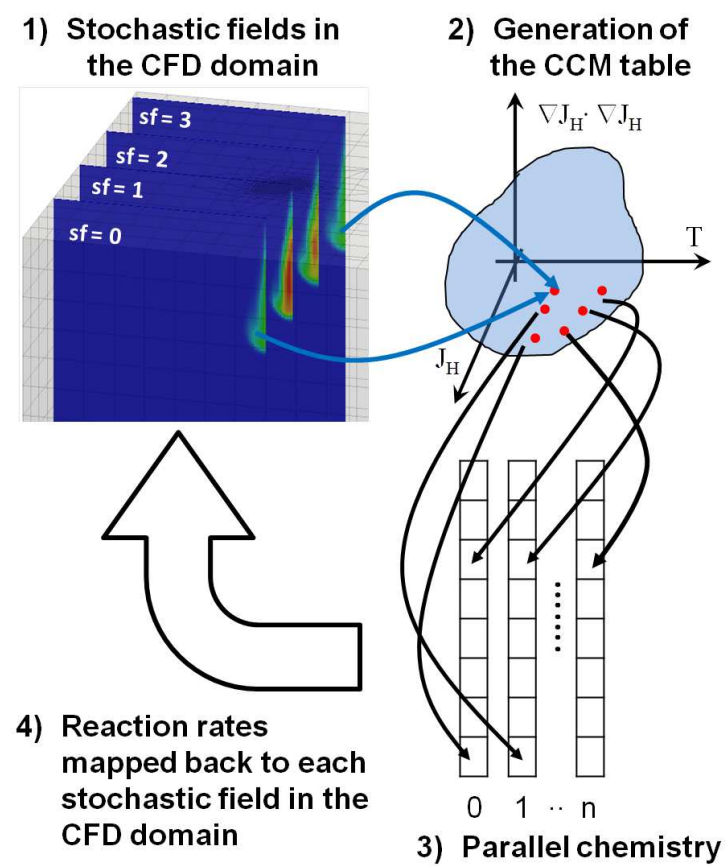
solution

Figure 1: Application of parallel Chemistry Coordinate Mapping technique (CCM) to chemistry integration with the SEF combustion model.

Accuracy of the CCM method was extensively verified by the authors in [31, 45] where it was applied to RANS and DNS non-premixed combustion simulations. To further reduce the CPU time for chemistry integration, the CCM method operates together with the TDAC algorithm [30, 32] which combines the ISAT and DAC techniques [41, 44, 48]. The ISAT algorithm intends to reuse computationally demanding results, e.g. the integration of large and stiff ODE systems, by storing those results and all the necessary data to retrieve them. The DAC method dynamically reduces the chemical mechanism is each cell and time-step before every call to the stiff solver according to the directed relation graph (DRG) method, which identifies the relevant species and reactions according to the thermodynamic conditions in each cell [44]. Combined operation of CCM, ISAT and DAC is schematically illustrated in Fig. 2. When ISAT receives from CCM a query $\psi^{q}$ that needs to integrate the ODE set, it provides $\psi^{q}$ to the DAC algorithm which then finds the reduced mechanism for the local thermochemical conditions and provides the reduced set of active species $\psi_{a}^{q}$ to the ODE solver. This solver computes the reaction mapping for the reduced set $\mathrm{R}\left(\psi_{a}^{q}\right)$ that is used by ISAT to build the reaction mapping $\mathbf{R}\left(\psi^{q}\right)$ in the full composition space. Using simplification methods at distinct levels combines their effects and allows a significant reduction of the computational cost. The use of TDAC ensures speed-up factors ranging from 10 to 1000 depending on the mechanism size and simulated combustion mode [32].

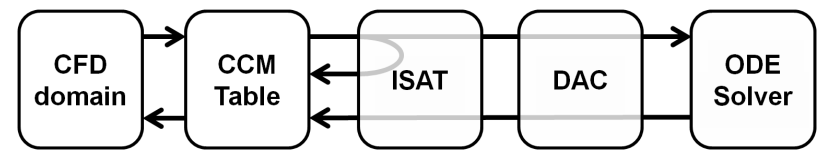

Figure 2: Combined operation of CCM, ISAT and DAC for acceleration of chemistry integration with SEF model.

\section{Experimental validation}

The main objective of the paper is understanding the performance of the SEF model when applied to combustion experiments at engine-like conditions, including spray evaporation and heat transfer. To properly describe all the physical and chemical processes, small time-steps are necessary and neither time-blending or under-relaxation are possible. Furthermore, both convergence and consistency of the method must be verified in presence of fuel evaporation. In particular, the Wiener term should not induce large mass and energy conservation errors during the fuel evaporation process and such errors must be rapidly reduced when increasing the total number of stochastic fields. Hence, application of the SEF method to the simulation of Diesel spray combustion is very challenging and a step-by-step validation is required in a first stage of the model assessment. Within this context, the following methodology was applied in this work:

1. simulation at non-reacting conditions, to understand the model capability to reproduce relevant global quantities, such as fuel mass in the domain. To this end, computed results were compared with the ones achieved by using a standard flow solver based on the well-stirred reactor assumption;

2. model capability to reproduce mixture fraction 
variance during simulations at non-reacting conditions.

3. comparison between predicted flame structure between SEF, well-stirred reactor and multiple representative interactive flamelet (mRIF) models.

\section{Sandia combustion vessel}

Experiments conducted within the Engine Combustion Network [49], http://www.ca.sandia.gov/ecn, in a constant-volume chamber were used to assess the potentialities of the SEF model to describe a turbulent spray flame. A single operating condition was considered, including both non-reacting and reacting conditions. The fuel used was n-dodecane $\left(\mathrm{nC}_{12} \mathrm{H}_{26}\right)$ and further details about the simulated ambient conditions are illustrated in Tab. 1.

Table 1: Simulated operating condition for Diesel combustion simulations.

\begin{tabular}{|c|c|}
\hline Ambient density & $22.8 \mathrm{~kg} / \mathrm{m}^{3}$ \\
\hline Ambient temperature & $900 \mathrm{~K}$ \\
\hline $\mathrm{O}_{2}$ concentration & $15 \%$ \\
\hline Injection pressure & $150 \mathrm{MPa}$ \\
\hline Nozzle diameter & $90 \mu \mathrm{m}$ \\
\hline Injection duration & $1.5 \mathrm{~ms}$ \\
\hline
\end{tabular}

Simulations were carried out in a 3D domain, using adaptive local mesh refinement [50] to reduce both grid dependency and computational time. In particular, the initial mesh size is $8 \mathrm{~mm}$ and then reduced to $0.5 \mathrm{~mm}$ only in regions where spray evolution and fuel-air mixing processes take place. A summary of the employed numerical set-up (e.g., mesh size and distribution, computational time step, numerical schemes, spray-sub model constants) is given in Tab. 2.

Table 2: Summary of the numerical setup used for spray combustion simulations.

\begin{tabular}{cc}
\hline Mesh type & 3D + ALMR \\
\hline \hline Minimum mesh size & $0.5 \mathrm{~mm}$ \\
\hline \hline Time step & $0.5 \mathrm{~ms}$ \\
\hline Atomization model & Huh-Gosman [51] \\
$\mathrm{C}_{5}$ & 1.5 \\
\hline Breakup model $_{1}$ & $\mathrm{KH}$ (wave) \\
$\mathrm{B}_{1}$ & 1.7 \\
\hline \hline Turbulence model & $k-\varepsilon$ \\
\hline \hline Mixing model coefficient $C_{\phi}$ & 2.0 \\
\hline Spatial discretizatoin & $2^{\text {nd }}$ order \\
\hline \hline Temporal discretizatoin & $1^{\text {st }}$ order \\
\hline
\end{tabular}

First, the validity of the proposed setup was verified at non reacting conditions in Fig. 3 , and a rather good agreement with experimental data was achieved for both computed liquid and vapor penetrations.

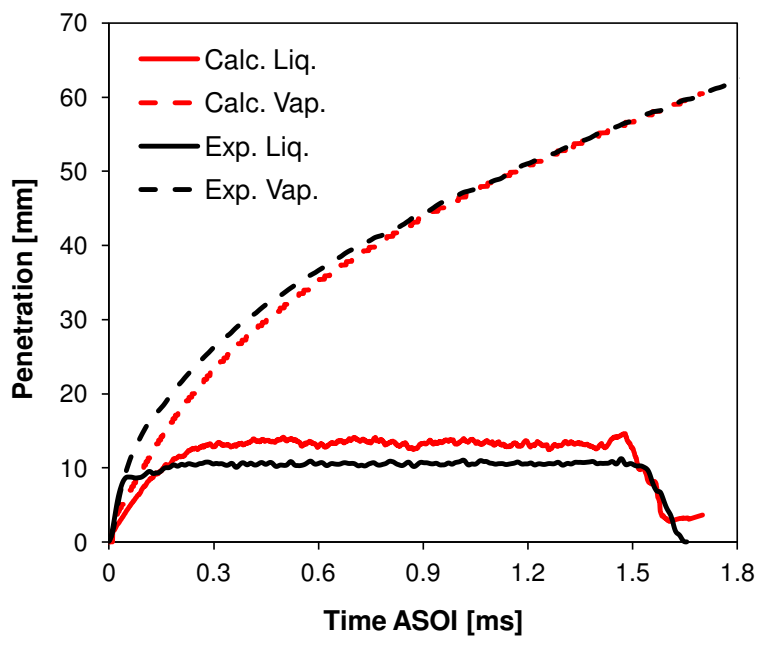

Figure 3: Spray model assessment: comparison between computed and experimental liquid and vapor penetrations.

Once the numerical setup was properly verified, non reacting conditions were analyzed and a sensitivity analysis was performed to understand the SEF model capability to conserve mass during the fuel injection phase. In particular, simulations were carried out with 4, 8, 16, 32 and 64 stochastic fields. In particular, computed fuel mass evolution from stochastic fields is compared with the one obtained directly from mixture fraction transport equation. Fuel mass from stochastic fields is computed as:

$$
m_{f u e l, S E F}(t)=\frac{1}{N_{f}} \sum_{i=1}^{N_{f}} \int_{V} \rho Y_{n C_{12} H_{26}, i} d v
$$

while computation of fuel mass from mixture fraction $Z$ requires an additional transport equation to be solved, including spray evaporation source term $(S)$ :

$$
\frac{\partial \rho \tilde{Z}}{\partial t}+\frac{\partial \rho \tilde{u}_{i} \tilde{Z}}{\partial x_{i}}-\frac{\partial}{\partial x_{i}}\left[\Gamma_{T Z} \frac{\partial Z}{\partial x_{i}}\right]=S
$$

The expected total fuel mass from $Z$ distribution is:

$$
m_{f u e l, Z}(t)=\int_{V} \rho Z d v
$$

Fig. 4(a) shows how the number of stochastic fields affects computed instantaneous fuel mass in the domain. Despite all the simulated setup are able to reproduce the proper trend with fuel mass continuously growing due to evaporation, severe discrepancies appear mainly for the cases where 4 and 8 fields are used. This is due to the fact that a small number of stochastic fields is not able to properly represent in each computational cell the proper PDF of the mixture 
fraction, and this creates inconsistencies between the fuel mass computed from stochastic fields compared with the ones deriving from mixture fraction equation. This aspect is further clarified by Fig. 4(b), which displays that the relative error between $m_{f u e l, S E F}$ and $m_{f u e l, Z}$ is very high towards the end of injection for the 8 stochastic fields case, while 4 stochastic fields produces very large error oscillations. Increasing the number of stochastic fields $\left(N_{F}>8\right)$ produces a better PDF and, consequently, a better agreement between $m_{f u e l, Z}$ and $m_{f u e l, S E F}$ is obtained, with instantaneous error remaining well below $3 \%$ for most of the injection duration.
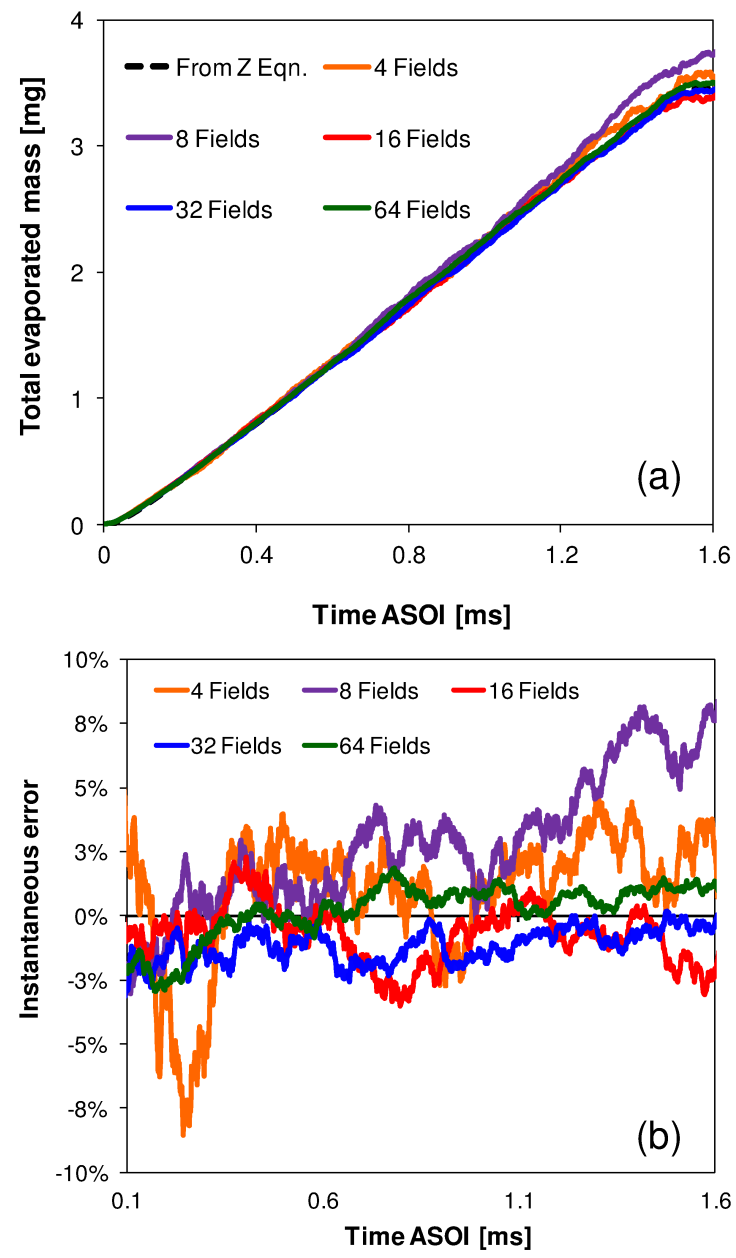

Figure 4: Effects of the number of stochastic fields on total instantaneous fuel vapor mass during the injection process. Results are reported from 4, 8, 16, 32 and 64 fields and fuel mass computed from mixture fraction distribution.

For sake of completeness, Tab. 3 reports how maximum instantaneous error and mean error are affected by the number of stochastic fields used: it is possible to see that the error is drastically reduced increasing them from 8 to 16 . Further increases do not substantially change the quality of results. The reason for this is related to the fact that, despite the number of stochastic fields is increased, it is still not possible to make the PDF of the mixture fraction completely regular and still some discontinuities in that appears. From this first investigation it is clear that at least 16 fields need to be used to consistently simulate the fuel-air mixing process.

Table 3: Effects of number of stochastic field on relative maximum and mean error in fuel mass compared to value calculated from mixture fraction distribution.

\begin{tabular}{|c|c|c|c|c|c|}
\hline & 4 & 8 & 16 & 32 & 64 \\
\hline Max err [\%] & 5.12 & 8.39 & 2.30 & 0.30 & 1.87 \\
\hline Mean err. [\%] & 0.96 & 2.63 & -0.74 & -1.17 & 0.12 \\
\hline
\end{tabular}
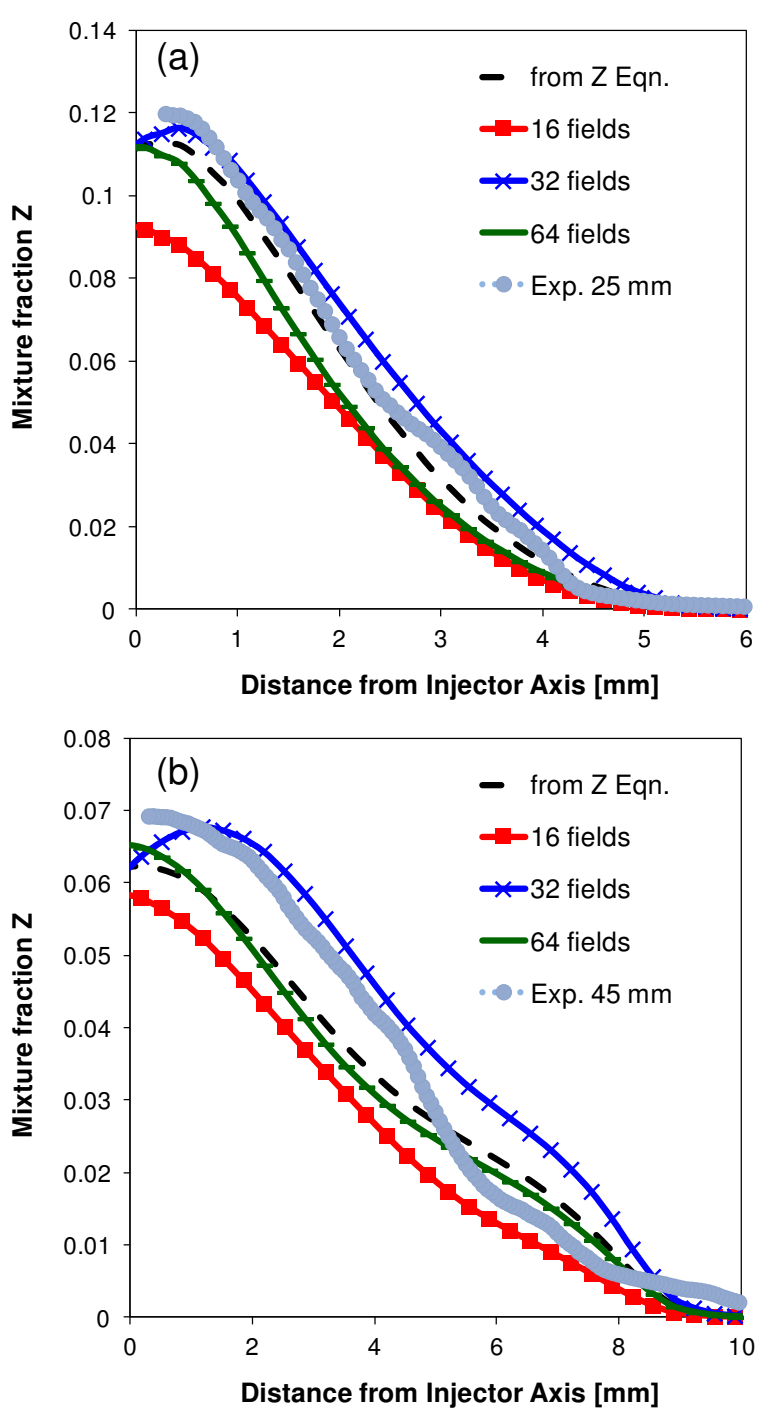

Figure 5: Comparison between computed and experimental radial distributions of mixture fraction: (a) $25 \mathrm{~mm}$ distance from injector; (b) $45 \mathrm{~mm}$ distance from injector. Computed data using 16, 32, 64 stochastic fields and mixture fraction transport equation.

The capability of the SEF model to reproduce experimental radial mixture fraction profiles is illustrated in 
Figs. 5(a)-(b), at two different distances (25 and 45 $\mathrm{mm}$ ) from the injector and $1.5 \mathrm{~ms}$ after start of injection (ASOI). Cases with 16, 32 and 64 stochastic fields were considered due to their acceptable results in terms of mass conservation. In both figures, also the measured and computed with Eq. 8 values are reported. For all the three displayed cases, results are in acceptable agreement with experimental data and, in particular, 64 stochastic fields seems to be necessary to reproduce the same symmetric profiles computed with the mixture fraction transport equation. When 32 stochastic fields are used, computed mixture fraction distribution looks non completely symmetric and slightly overestimating experimental data. 16 stochastic fields underpredict radial profiles and, despite not shown here, exhibit a non-negligible asymmetry with respect to the injector axis.

Very similar considerations can be done for what concerns the predicted axial mixture fraction distribution which is shown in Fig. 6 . Both 32 and 64 fields agree rather well with both experimental data and distribution computed from mixture fraction transport equation. Again, results with 16 stochastic underestimates experimental data and are not completely axysymmetric.

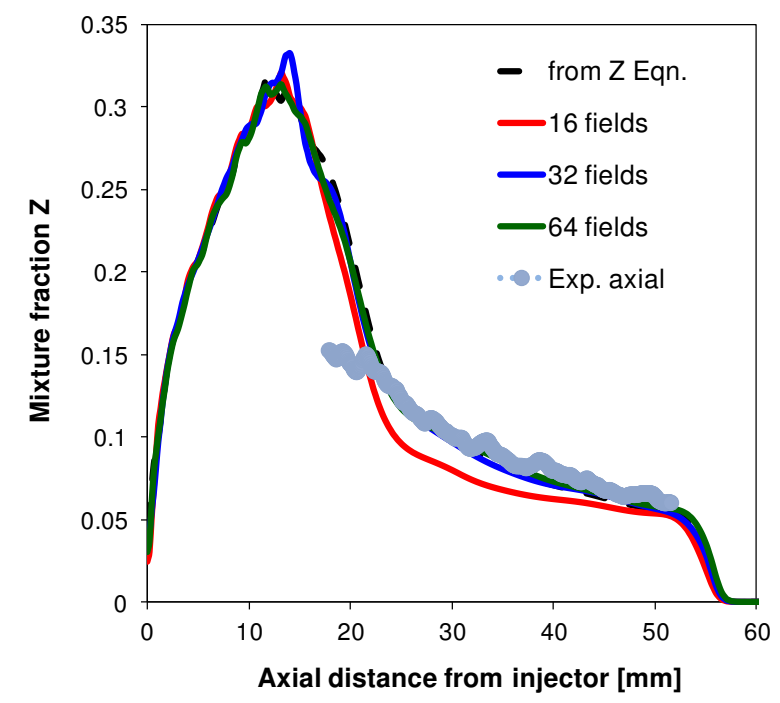

Figure 6: Comparison between computed and experimental axial distributions of mixture fraction. Computed data using $16,32,64$ stochastic fields and mixture fraction transport equation.

In the SEF model, the capability to reproduce the PDF of the main species makes possible to use chemical source terms in a closed form in both species and energy equations. For this reason, it is necessary to verify the model capability to reproduce variances of the main species since they strongly affect heat release during the combustion process. Here, a comparison between computed mixture fraction variance from stochastic fields and one computed from trans- port equation according to [52] was performed. SEF mixture fraction variance $\widetilde{Z^{\prime \prime 2}} S_{S E F}$ was defined as:

$$
{\widetilde{Z^{\prime \prime 2}}}_{S E F}=\frac{1}{N_{f}} \sum_{i=1}^{N_{f}}\left(Y_{n C_{12} H_{26}, i}-\tilde{Y}_{c C_{12} H_{26}}\right)^{2}
$$

while formulation proposed by [52] was used to compute mixture fraction variance $\widetilde{Z^{\prime \prime 2}}$ with the following transport equation:

$$
\begin{gathered}
\frac{\partial \rho \widetilde{Z^{\prime \prime 2}}}{\partial t}+\frac{\partial \rho \tilde{u}_{i} \widetilde{Z^{\prime \prime 2}}}{\partial x_{i}}-\frac{\partial}{\partial x_{i}}\left[\Gamma_{T \widetilde{Z^{\prime \prime 2}}} \frac{\partial \widetilde{Z^{\prime \prime 2}}}{\partial x_{i}}\right]= \\
2 \Gamma_{T \widetilde{Z^{\prime \prime 2}}}(\nabla Z \cdot \nabla Z)-\rho \chi
\end{gathered}
$$

where $\chi$ is the scalar dissipation rate, defined as:

$$
\chi=C_{\chi} \widetilde{Z^{\prime \prime 2}} \omega
$$

Following $[52,13,53]$, the $C_{\chi}$ term was set to 2.0 .

Figs. 7(a)-(b) report computed radial distributions of mixture fraction variance at 25 and $45 \mathrm{~mm}$ distances from the injector axis using 16, 32 and 64 stochastic fields. In the same figures, also computed data with Eq. 11 and experimental profiles are reported. It is interesting to see that SEF method and Eq. 11 predicts values of the same order of magnitude without any tuning of both $C_{\chi}$ and $C_{\phi}$ constants for mixing. Results from the SEF method, at least with 32 and 64 stochastic fields are in rather good agreement with experimental data mainly at $25 \mathrm{~mm}$ distance from injector, while both 16 fields and $\widetilde{Z^{\prime \prime} 2}$ from Eq. 11 overestimate the experimental values. A good prediction of mixture fraction variance at this position might positively affect the computed lift-off length which is experimentally found $18 \mathrm{~mm}$ far from the injector. Further downstream, a rather good agreement between computed and experimental data was achieved except for the 16 stochastic field case, still overestimating the experimental distribution.

For what concerns the axial distribution of the mixture fraction variance, close to the nozzles, up to 15 from it, $\widetilde{Z^{\prime \prime 2}}$ computed from stochastic fields is quite lower than values estimated with Eq. 11, even if such equation does not include effects of spray evaporation as suggested in [54]. Further downstream, a rather good agreement was found and all the tested configurations showing almost the same hump at the fuel vapor tip. Results achieved so far with the SEF method applied to fuel-air mixing process for diesel spray are 
very promising and allowed to identify that with almost 32 stochastic fields it was possible to predict mixture fraction and its variance distribution very accurately.
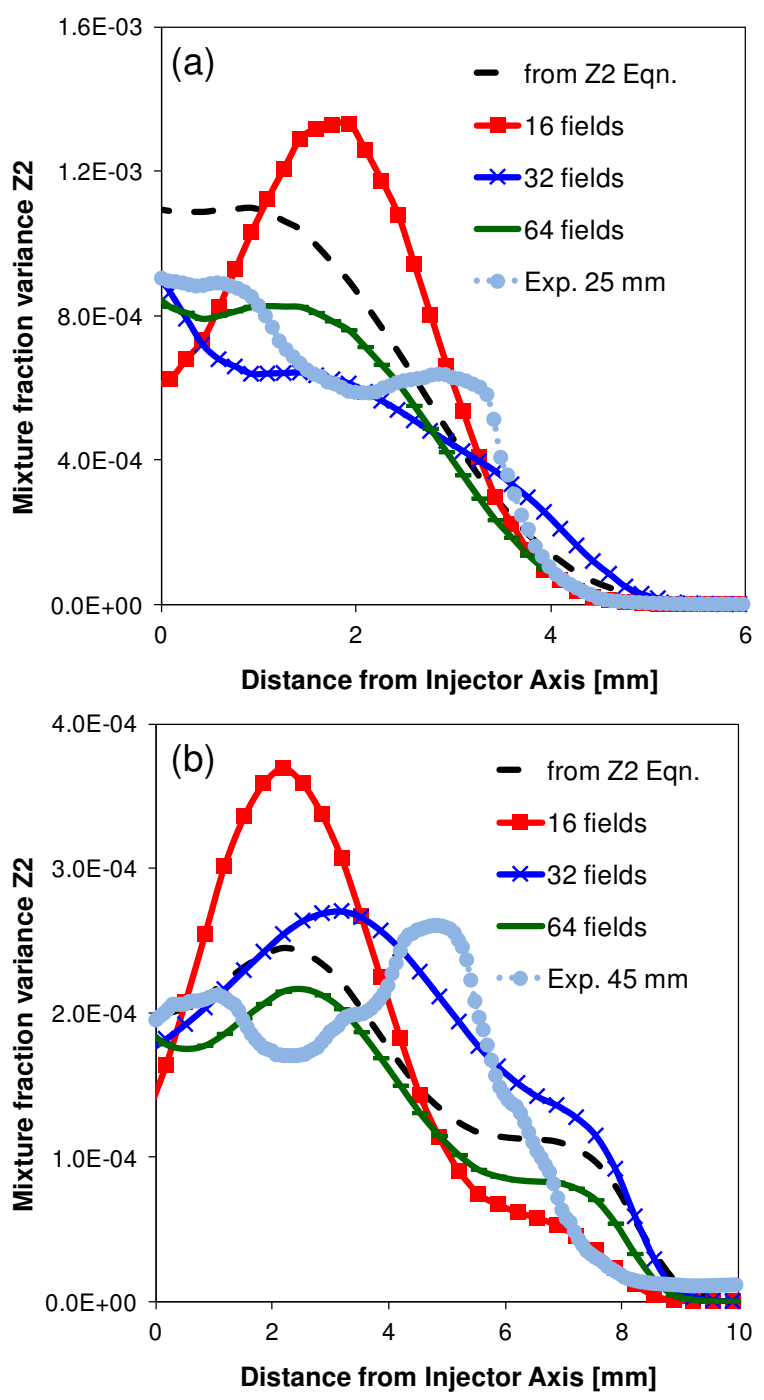

Figure 7: Comparison between computed and experimental radial distributions of mixture fraction variance: (a) 25 $\mathrm{mm}$ distance from injector; (b) $45 \mathrm{~mm}$ distance from injector. Computed data using 16, 32, 64 stochastic fields and mixture fraction transport equation.

Once consistency and convergence of the method was verified at non-reacting conditions, combustion simulations were performed. A reduced mechanism for $n$-dodecane proposed in [55] was used. It has 106 species and 420 reactions and it was extensively validated with constant-volume ignition delay data in a wide range of ambient conditions, including variation of pressure, ambient temperature and equivalence ratio. Preliminary results are presented in this work, in particular results from the SEF model are compared with the ones achieved by the well-mixed and mRIF model $[13,14]$. Both these last ones were recently implemented in the Lib-ICE code and extensively validated in $[56,57]$ where the reader can refer for fur-

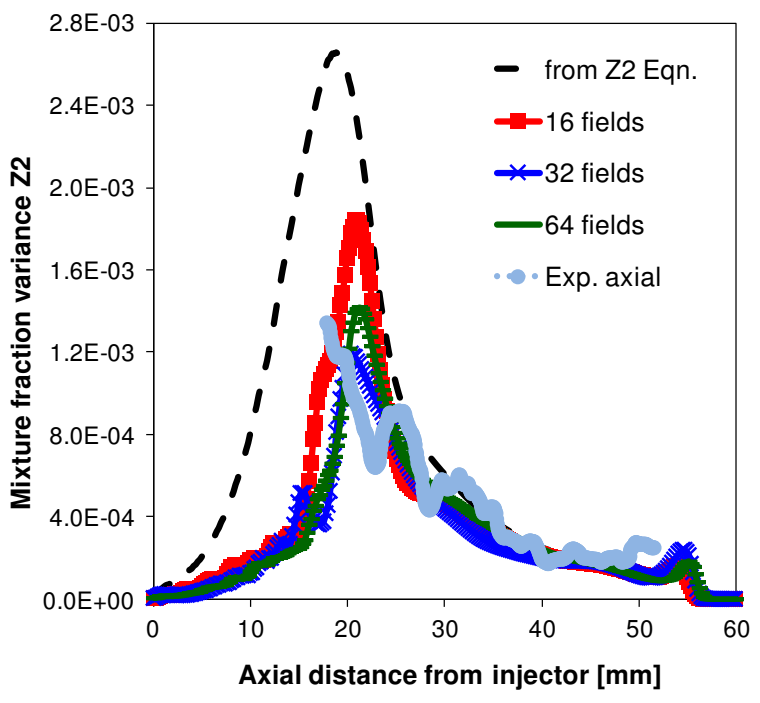

Figure 8: Comparison between computed and experimental axial distributions of mixture fraction variance. Computed data using 16, 32, 64 stochastic fields and mixture fraction transport equation.

ther information. In the mRIF simulations, 15 flamelets were used: they are sequentially created during injection and each one of them includes $1 / 15$ of the total injected fuel mass. For what concerns simulations with the SEF model, despite it was shown that at least 32 stochastic fields were necessary to achieve convergence in mass conservation, results with 16 fields are presented here. In a future work, also a sensitivity analysis at reacting conditions will be performed. For all the three models, auto-ignition takes place at approximately $0.6 \mathrm{~ms}$, while experimentally this was found at $0.44 \mathrm{~ms}$. Since such discrepancy was consistently predicted by all the tested models, authors think this was mainly due to the kinetic mechanism used. A qualitative comparison in terms of flame structure is provided in Figs. 9(a)-(b) showing temperature field, fuel mass fraction contours (in white) and $10^{-4} \mathrm{OH}$ mass fraction iso-contour with a black line. Fig. 9(a) displays results at $0.45 \mathrm{~ms}$ after SOI, immediately after cool flame ignition delay. At this time, it is possible to see that all three models predicts maximum temperatures which are just slightly higher than the ambient one. However, the way cool ignition is predicted is quite different for all them. The well-mixed model predicts a cool flame mainly located at the bottom of the fuel vapor region, while cool flame region from SEF model is larger due to a better way to represent the PDF of the reacting chemical species. The mRIF model predicts lower peak temperatures at $0.45 \mathrm{~ms}$ with a large cool-flame zone and this is mainly related to the model assumptions of representing non-premixed combustion with a set of $1 \mathrm{D}$, unsteady flamelets and the use of a $\beta$-PDF to estimate species concentration in each cell. 
(a) Well-mixed

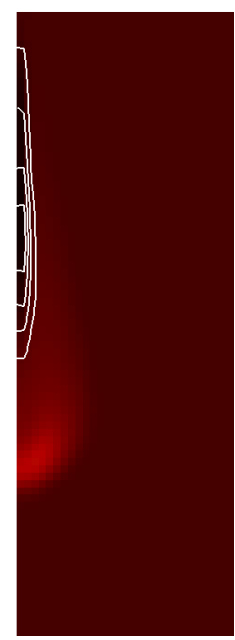

(b) Well-mixed
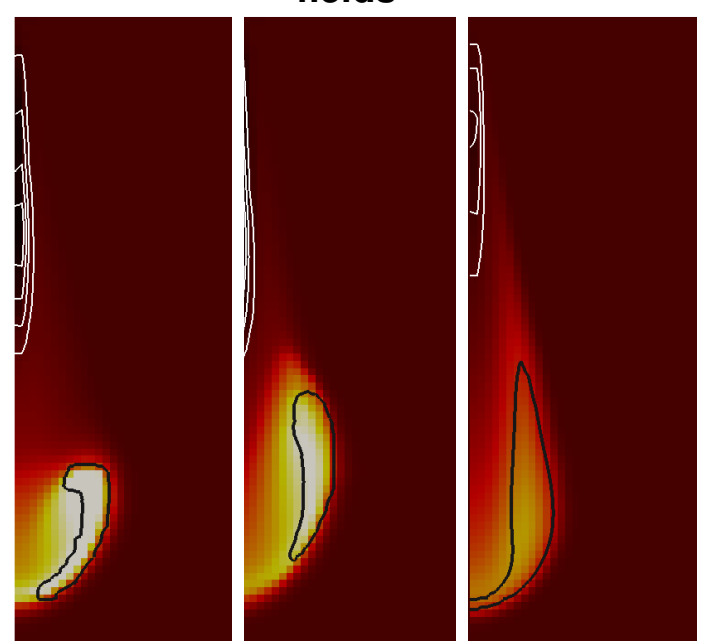

Figure 9: Comparison between computed flame structures from well-mixed, SEF (with 16 stochastic fields) and mRIF (with 15 flamelets) models. Temperature distribution: scale 700 (red) - 2000 (white); fuel mass fraction iso-contours (white, range is $0-0.02) ; \mathrm{OH}$ mass fraction iso-contour (black line correspond to $10^{-4}$ mass fraction. (a) flame structure at $0.45 \mathrm{~ms}$ after $\mathrm{SOI}$; (b) flame structure at 0.7 ms after SOI.

The flame stabilizes soon after auto-ignition, and Fig. 9 reports its structure at $0.7 \mathrm{~ms}$ after SOI computed by the three different models. Despite similar temperature fields were found at auto-ignition time, computed flame shapes are rather different at $0.7 \mathrm{~ms}$ and this is due to the different way the employed models stabilize the flame. When the well-mixed assumption is used, stabilization is mainly governed by turbulent diffusion and local flow conditions [57] which determine a thin region where most of heat release takes place. In the mRIF model, auto-ignition of multiple diffusion flames was found to be the stabilization mechanism $[57,56]$, and for this reason the flame is much longer and, due to turbulence chemistry interaction, even wider. The flame structure from SEF is different from the other two models. First, the PDF of the chemical species is mainly created due to the Wiener term in the core region of the spray, where fuel evaporates and turbulent diffusion is very high. Large variances will be then smoothed downstream due to turbulent mixing. Hence, in the SEF model a diffusion flame structure is first created and, after autoignition, turbulent mixing determines the temperature fields where heat release takes place. For what concerns flame stabilization, surely it is affected by local flow and diffusion but also turbulence mixing is also expected to play a role there. As a consequence of this, the SEF model seems to incorporate aspects of both well-mixed and mRIF models and for this reason it might be a very powerful tool for combustion modelling. However, further investigations are required to better understand its performance at reacting conditions.

\section{Conclusions}

Purpose of this paper was the assessment of a Stochastic Eulerian Field combustion model to be applied for IC engine simulations. Advantages of such model are mainly represented by its flexibility in terms of combustion modes to be simulated since no subgrid model or flame structure assumption are necessary to compute the chemical species reaction rate. The approach originally proposed by Valiño [25] was followed in this work and implemented into the LibICE code. Combustion experiments carried out in the SANDIA constant volume vessel were used to validate the proposed approach [49]. Such configuration was mainly chosen due to the large amount of related documentation and measured data. Initially the SEF model capability to conserve global quantities was verified. It was found that when more than 32 fields were used, mass conservation errors become relatively small. The use of less stochastic fields, on the other hands, does not allow to compute the correct amount of fuel mass, due to the too small number of points available for the reconstruction of mixture fraction PDF. The SEF model was also able to properly reproduce mixture fraction variance distributions inside the whole domain. This is expected to be very important to describe both auto-ignition and combustion processes. Finally, preliminary analyses carried out at reacting conditions shows that the SEF model is able to predict both premixed and non-premixed features in the flame structure, making it very promising for turbulent combustion simulations. Further work is required to include a comprehensive validation at different ambient conditions and investigate if it is possible to reduce the number of stochastic fields required 
for the statistical convergence.

\section{CONTACT INFORMATION}

\author{
Dr. Tommaso Lucchini \\ Department of Energy, Politecnico di Milano \\ Via Lambruschini, 4 \\ 20156 Milano, Italy \\ tommaso.lucchini@polimi.it
}

\section{References}

1. M. Sjoberg and J. E. Dec. Smoothing HCCl heatrelease rates using partial fuel stratification with two-stage ignition fuels. SAE Paper, 2006-010629, 2006.

2. H. Zhao, Z. Peng, J. Williams, and N. Ladommatos. Understanding the Effects of Recycled Burnt Gases on the Controlled Autoignition (CAI) Combustion in Four-Stroke Gasoline Engines. SAE Paper, 2001-01-3607, 2001.

3. H. Persson, A. Hultqvist, B. Johansson, and A. Remon. Investigation of the Early Flame Development in Spark Assisted $\mathrm{HCCl}$ Combustion Using High Speed Chemiluminescence Imaging. SAE Paper, 2007-01-0212, 2007.

4. F. Atzler, O. Kastner, R. Rotondi, and A. Weigand. Multiple injection and rate shaping Part 1: Emissions reduction in passenger car Diesel engines. SAE Paper, 2009-24-0004, 2009.

5. C. Stan, S. Guenther, L. Martorano, and C. Tarantino. Aspects of Mixture Formation and Combustion in GDI Engines. SAE Paper, 200001-0648, 2000.

6. Z. Han, C. Weaver, S. Wooldridge, and T. Alger et al. Development of a New Light StratifiedCharge DISI Combustion System for a Family of Engines With Upfront CFD Coupling With Thermal and Optical Engine Experiments. SAE Paper, 2004-01-0545, 2004.

7. A. Kultzer, J. Hathout, C. Sauer, R. Karrelmeyer, W. Fischer, and A. Christ. Multi-Mode Combustion Strategies with CAI for a GDI Engine. SAE Paper, 2007-01-0214, 2007.

8. V. Mittal, D. J. Cook, and D. Pitsch. An extended multi-regime flamelet model for $\{$ IC $\}$ engines. Combustion and Flame, 159(8):2767 2776, 2012.

9. P. K. Senecal, K. J. Richards, E. Pomraning, T. Yang, M. Z. Dai, R. M. McDavid, M. A. Pat- terson, S. Hou, and T. Shethaji. A New Parallel Cut-Cell Cartesian CFD Code for Rapid Grid Generation Applied to In-Cylinder Diesel Engine Simulations. SAE Paper, 2007-01-0159, 2007.

10. S. Singh, R. D. Reitz, and M. P. B. Musculus. Comparison of the Characteristic Time (CTC), Representative Interactive Flamelet (RIF), and Direct Integration with Detailed Chemistry Combustion Models against Optical Diagnostic Data for Multi-Mode Combustion in a Heavy-Duty DI Diesel Engine. SAE Paper, 2006-01-0055, 2006.

11. T. Lucchini, G. D'Errico, D. Ettorre, and G. Ferrari. Numerical investigation of non-reacting and reacting Diesel sprays in constant-volume vessels. SAE paper, 2009-01-1971, 2009.

12. S. Som and S.K. Aggarwal. Effects of primary breakup modeling on spray and combustion characteristics of compression ignition engines. Combustion and Flame, 157(6):1179 - 1193, 2010.

13. H. Barths, C. Hasse, and N. Peters. Computational fluid dynamics modelling of non-premixed combustion in direct injection diesel engines. International Journal of Engine Research, 1 (3):pp. 249-267, 2000.

14. H. Lehtiniemi, Y. Zhang, R. Rawat, and F. Mauss. Efficient 3-D CFD Combustion Modeling with Transient Flamelet Models. SAE Paper, 2008-010957, 2008.

15. Y. M. Wright, K. Boulouchos, G. De Paola, and E. Mastorakos. Multi-dimensional Conditional Moment Closure Modelling Applied to a Heavyduty Common-rail Diesel Engine. SAE Paper, 2009-01-0717, 2009.

16. YM Wright, $G$ De Paola, $K$ Boulouchos, and E Mastorakos. Simulations of spray autoignition and flame establishment with two-dimensional cmc. Combustion and flame, 143(4):402-419, 2005.

17. D. Farrace, M. Bolla, Y. Wright, and K. Boulouchos. Predicting In-Cylinder Soot in a Heavy-Duty Diesel Engine for Variations in SOI and TDC Temperature Using the Conditional Moment Closure Model. SAE Paper, 2013-24-0016, 2013.

18. S. B. Pope. PDF methods for turbulent reactive flows. Progress in Energy and Combustion Science, Vol. 11:pp. 119-192, 1985.

19. S. B. Pope. Turbulent Flows. Cambridge University Press, 2000.

20. R. O. Fox. Computational Models for Turbulent Reacting Flows. Cambridge University Press, 2003. 
21. D. C. Haworth. Progress in probability density function methods for turbulent reacting flows. Progress in Energy and Combustion Science, Vol. 36:pp. 168259, 2010.

22. D.C. Haworth and S.B. Pope. Transported probability density function methods for Reynoldsaveraged and large-eddy simulations. in Turbulent Combustion Modeling: Advances, New Trends and Perspectives, T. Echekki and E. Mastorakos, Springer, 2011.

23. E. Kung and D. C. Haworth. Transported Probability Density Function (tPDF) Modeling for DirectInjection Internal Combustion Engines. SAE Int. J. Engines, 1 (1):pp. 591-606, 2009.

24. C. Taut, C. Correa, O. Deutschmann, J. Warnatz, S. Einecke, C. Schulz, and J. Wolfrum. ThreeDimensional Modeling With Monte CarloProbability Density Function Methods And Laser Diagnostics Of The Combustion In A Two-Stroke Engine. Proceedings of the Combustion Institute, Vol. 28:pp. 1153-1159, 2000.

25. L. Vali no. A field Monte Carlo formulation for calculating the probability density function of a single scalar in a turbulent flow. Flow, Turbul. Combust., 60:pp. 157-172, 1998.

26. S. Bhattacharjee, J. Jaishree, V. Raj Mohan, H. Zhang, and D.C. Haworth. PDF-based simulations of turbulent spray combustion in a constantvolume chamber under diesel-engine-like conditions. Proceedings of the International Multidimensional Engine Modeling Users Group Meeting, 2012.

27. Vinayaka N. Prasad, Assaad R. Masri, Salvador Navarro-Martinez, and Kai H. Luo. Investigation of auto-ignition in turbulent methanol spray flames using large eddy simulation. Combustion and Flame, 160(12):2941 - 2954, 2013.

28. J. Jaishree and D.C. Haworth. Comparisons of Lagrangian and Eulerian PDF methods in simulations of non-premixed turbulent jet flames with moderate-to-strong turbulence-chemistry interactions. Combustion Theory and Modelling, Vol. 16:pp. 435463, 2012.

29. W.P. Jones and V.N. Prasad. Les-pdf simulation of a spark ignited turbulent methane jet. Proceedings of the Combustion Institute, 33(1):1355 $-1363,2011$.

30. F. Contino, H. Jeanmart, T. Lucchini, and G. DErrico. Coupling of in situ adaptive tabulation and dynamic adaptive chemistry: An effective method for solving combustion in engine simulations.
Proceedings of the Combustion Institute, Vol. 33(2):pp. 3057-3064, 2011.

31. M. Jangi, R. Yu, and X. S. Bai. A multi-zone chemistry mapping approach for direct numerical simulation of auto-ignition and flame propagation in a constant volume enclosure. Combustion Theory and Modelling, 16(2):221-249, 2012.

32. F. Contino, T. Lucchini, G. D'Errico, C. Duynslaegher, V. Diaz, and H. Jeanmart. Simulations of Advanced Combustion Modes Using Detailed Chemistry Combined with Tabulation and Mechanism Reduction Techniques. SAE Int. J. Engines, 5:185-196, 2012.

33. J. Villermaux and J.C. Devillon. Representation de la coalescence et de la redispersion des domaines de segregation dans un fluide par un modele dinteraction phenomenologique. in Proceedings of Second International Symposium on Chemical Reaction Engineering, pages pp. 1-13, 1972.

34. J. Wallesten. Micromixing effects in atmospheric reacting flows. Phd thesis, University of Cambridge, 2007.

35. J. Stoer and F. Bulirsch. Introduction to Numerical Analysis. Springer-Verlag, 1980.

36. C. Bekdemir, L.M.T. Somers, and L.P.H. de Goey. Modeling diesel engine combustion using pressure dependent flamelet generated manifolds. Proceedings of the Combustion Institute, 33(2):2887 - 2894, 2011.

37. F. Tap and D. Veynante. Simulation of Flame Liftoff on a Diesel Jet Using a Generalized Flame Surface Density Approach. Proceedings of the Combustion Institute, 30:pp. 919-926, 2005.

38. Phuc-Danh Nguyen, Luc Vervisch, Vallinayagam Subramanian, and Pascale Domingo. Multidimensional flamelet-generated manifolds for partially premixed combustion. Combustion and Flame, 157(1):43 - 61, 2010.

39. R. Aglave, U. Riedel, and J. Warnatz. TurbulenceChemistry Interactions in CFD Modelling of Diesel Engines. Combustion Theory and Modelling, 12(2):pp. 305-328, 2008.

40. P.K. Senecal, D.P. Schmidt, I. Nouar, C.J. Rutland, R.D Reitz, J.K. Martin, and J.A. Hoffman. Pressure-Swirl Atomization in the Near Field. SAE Paper, 1999-01-0496, 1999.

41. M.A. Singer and S.B. Pope. Exploiting ISAT to solve the equations of reacting flow. Combustion Theory and Modelling, Vol. 8:pp. 361-383, 2004. 
42. O. T. Stein, G. Olenik, A. Kronenburg, F. Cavallo Marincola, B. M. Franchetti, A. M. Kempf, M. Ghiani, M. Vascellari, and C. Hasse. Towards comprehensive coal combustion modelling for les. Flow, Turbulence and Combustion, 90(4):859884, 2013.

43. S. B. Pope. Computationally Efficient Implementation of Combustion Chemistry Using In-Situ Adaptive Tabulation. Combustion Theory and Modelling, 1:pp. 14-63, 1997.

44. Long Liang, John G. Stevens, Sumathy Raman, and John T. Farrell. The use of dynamic adaptive chemistry in combustion simulation of gasoline surrogate fuels. Combustion and Flame, 156(7):1493 - 1502, 2009.

45. Mehdi Jangi, Tommaso Lucchini, Gianluca DErrico, and Xue-Song Bai. Effects of egr on the structure and emissions of diesel combustion. Proceedings of the Combustion Institute, 34(2):3091 - 3098, 2013.

46. M. Raju, M. Wang, M. Dai, W. Piggott, and D. Flowers. Acceleration of Detailed Chemical Kinetics Using Multi-zone Modeling for CFD in Internal Combustion Engine Simulations. SAE Paper, 2012-01-0135, 2012.

47. A. Babajimopoulos, D. N. Assanis, D. L. Flowers, S. M. Aceves, and R. P. Hessel. A fully coupled computational fluid dynamics and multizone model with detailed chemical kinetics for the simulation of premixed charge compression ignition engines. International Journal of Engine Research, Vol. 6:497-512, 2005.

48. Y. Shi, L. Liang, H.-W. Ge, and R. D. Reitz. Acceleration of the chemistry solver for modeling di engine combustion using dynamic adaptive chemistry (dac) schemes. Combustion Theory and Modelling, 14(1):69-89, 2010.

49. M. Meijer, B. Somers, J. Johnson, J. Naber, S.Y. Lee, L. M. Malbec, G. Bruneaux, L. M. Pickett, M. Bardi, R. Payri, and T. Bazyn. Engine combustion network (ecn): Characterization and comparison of boundary conditions for different combustion vessels. Atomization and Sprays, 22(9):777806, 2012.

50. T. Lucchini, G. D’Errico, and D. Ettorre. Numerical investigation of the spraymeshturbulence interactions for high-pressure, evaporating sprays at engine conditions. International Journal of Heat and Fluid Flow, 32(1):285 - 297, 2011.
51. K. Y. Huh and A. D. Gosman. A Phenomenological Model of Diesel Spray Atomization. Proceedings of the International Conference on Multiphase Flows, Tsukuba, Japan, 1991.

52. H. Pitsch, H. Barths, and N. Peters. Three Dimensional Modeling of NOx and Soot Formation in DIDiesel Engines Using Detailed Chemistry Based on the Interactive Flamelet Approach. SAE Paper 962057, SAE Transactions, 105(4):2010-2025, 1996.

53. C. Hasse. A Two-Dimensional Flamelet Model for Multiple Injections in Diesel Engines. PhD thesis, Technischen Hochschule Aachen, 2004.

54. J. Rveillon and L. Vervisch. Spray vaporization in nonpremixed turbulent combustion modeling: a single droplet model. Combustion and Flame, 121(12):75 - 90, 2000.

55. S. Som, D.E. Longman, Z. Luo, M. Plomer, and T. Lu. Three Dimensional simulations of diesel sprays using n-dodecane as a surrogate. In Eastern States Section of the Combustion Institute Fall Technical Meeting, 2011.

56. G. D’Errico, T. Lucchini, A. Stagni, A. Frassoldati, T. Faravelli, and E. Ranzi. Reduced Kinetic Mechanisms for Diesel Spray Combustion Simulations. SAE Paper, 2013-24-0014, 2013.

57. G. D’Errico, T. Lucchini, F. Contino, M. Jangi, and X-S. Bai. Comparison of well-mixed and multiple representative interactive flamelet approaches for Diesel spray combustion modelling. Combustion Theory and Modelling, page In press, 2014. 